\title{
Advanced Ultrasound Technologies for Diagnosis and Therapy
}

\author{
Anne Rix ${ }^{1}$, Wiltrud Lederle ${ }^{1}$, Benjamin Theek ${ }^{1}$, Twan Lammers ${ }^{1,2}$, Chrit Moonen ${ }^{3}$, Georg Schmitz ${ }^{4}$, and Fabian Kiessling ${ }^{1}$ \\ ${ }^{I}$ Institute for Experimental Molecular Imaging, RWTH-Aachen University, Aachen, Germany; ${ }^{2}$ Department of Targeted Therapeutics, \\ University of Twente, Enschede, The Netherlands; ${ }^{3}$ Imaging Division, University Medical Center Utrecht, Utrecht, The Netherlands; \\ and ${ }^{4}$ Department of Medical Engineering, Ruhr-University Bochum, Bochum, Germany
}

\begin{abstract}
Ultrasound is among the most rapidly advancing imaging techniques. Functional methods such as elastography have been clinically introduced, and tissue characterization is improved by contrast-enhanced scans. Here, novel superresolution techniques provide unique morphologic and functional insights into tissue vascularization. Functional analyses are complemented by molecular ultrasound imaging, to visualize markers of inflammation and angiogenesis. The full potential of diagnostic ultrasound may become apparent by integrating these multiple imaging features in radiomics approaches. Emerging interest in ultrasound also results from its therapeutic potential. Various applications of tumor ablation with highintensity focused ultrasound are being clinically evaluated, and its performance strongly benefits from the integration into MRI. Additionally, oscillating microbubbles mediate sonoporation to open biologic barriers, thus improving the delivery of drugs or nucleic acids that are coadministered or coformulated with microbubbles. This article provides an overview of recent developments in diagnostic and therapeutic ultrasound, highlighting multiple innovation tracks and their translational potential.
\end{abstract}

Key Words: drug delivery; HIFU; molecular imaging; superresolution; ultrasound

J Nucl Med 2018; 59:740-746

DOI: 10.2967/jnumed.117.200030

$\mathbf{U}$

ltrasound is among the most frequently used imaging modalities in the clinic. However, in comparison to other tomographic imaging modalities, it is less reproducible because identical image slices are difficult to regain at repetitive examinations. Furthermore, the skills and experience of the examining physician have a strong impact on its diagnostic reliability. These limitations may be overcome by the implementation of truly 3-dimensional (3D) imaging, automated image analysis, and postprocessing tools, as well as by further standardization of scan protocols and data documentation. Solving these issues may also stimu-

Received Nov. 30, 2017; revision accepted Feb. 20, 2018.

For correspondence or reprints contact: Fabian Kiessling, Institute for Experimental Molecular Imaging, University Aachen (RWTH), Forckenbeckstrasse 55, 52074 Aachen, Germany.

E-mail: fkiessling@ukaachen.de

Published online Mar. 1, 2018.

COPYRIGHT (C) 2018 by the Society of Nuclear Medicine and Molecular Imaging. late the clinical implementation of emerging ultrasound technologies and contrast agents for diagnosis and therapy, which were discussed in our previous article in 2012 (1). At that time, most examples were related to preclinical studies. Since then, much clinical translation has occurred, such as in contrast-enhanced functional and molecular ultrasound imaging, focused ultrasound, and sonoporation. The present article highlights recent technologic advancements in ultrasound diagnosis and therapy that are in the process of clinical translation and are strongly expected to shape the future clinical appearance of this modality.

\section{NEW ULTRASOUND CONTRAST METHODS}

The contrast in ultrasound grayscale imaging originates mainly from variations in tissue compressibility and density. However, imaging methods in current ultrasound systems cannot reconstruct absolute material parameters directly from the received echo signals. In principle, this reconstruction is possible with full-wavefield inversion techniques, even with conventional ultrasound array setups, but the computational burden of these methods is still too high for real-time imaging (2).

Elastography methods that can image the mechanical properties of tissue have been developed in the last 2 decades, first as strain imaging techniques using external sources of deformation, and then by line-based measurements of shear-wave speed using no external compression but acoustic radiation force imaging. Later, fully 2dimensional shear-wave imaging (Fig. 1A) based on ultrafast plane-wave acquisitions with repetition rates in the kilohertz range was added: by observing shear waves traveling at low speeds $(1-10 \mathrm{~m} / \mathrm{s})$ with high-frame-rate plane-wave ultrasound acquisitions, the local shear-wave speed can be measured. This modality allows clinical imaging of the shear-wave speed or the shear modulus of tissues (3). Because the shear waves are excited by the acoustic radiation force induced by a focused ultrasound pulse, a major advantage of this technique is that it is less operatordependent than strain imaging techniques relying on manual deformation. The main clinical applications of the technique are diagnosis of liver fibrosis and characterization of breast and thyroid tumors (Fig. 1B). However, the size and location of the lesion, the composition of the surrounding tissue, and motion artifacts from breathing or pulsatile 


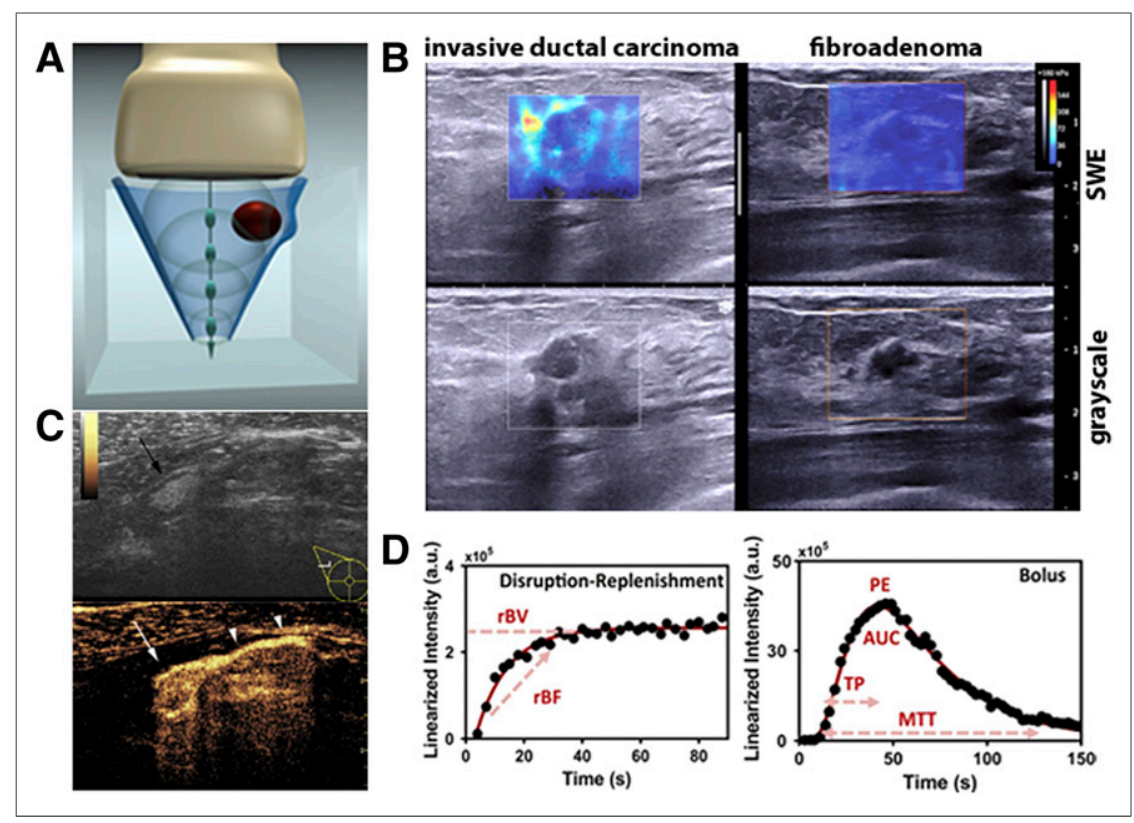

FIGURE 1. (A) Principle of shear-wave elastography: push pulses with 4 different foci generate shear waves that superimpose with shear-wave cone. Lesion (red) with different shear-wave speed deforms wavefront, as observed with high-frame-rate plane-wave imaging. (B) Shear-wave elastography and grayscale images of grade III invasive ductal carcinoma with maximum elasticity of $153 \mathrm{kPa}$ and fibroadenoma with homogeneously low elasticities with maximum of $21.7 \mathrm{kPa}$ (Reproduced with permission of (3).) (C) Microbubble-enhanced sentinel lymph node imaging. Representative grayscale image of axillary lymph node basin shows lymph node with fatty hilum. Corresponding CEUS image indicates connection of enhanced lymph node to lymphatic vessel. (Reproduced from (11); licensed by Creative Commons-Attribution 4.0 International.) (D) CEUS. Graphs show several functional parameters that can be obtained from disruptionreplenishment and bolus time-intensity curves. AUC = area under the curve; $P E=$ peak enhancement; $\mathrm{MTT}=$ mean transit time; $\mathrm{rBF}=$ relative blood flow; $\mathrm{rBV}=$ relative blood volume; TP = time to peak. (Adapted from (13); licensed by Creative Commons-Attribution 4.0 International.)

vessels limit the accuracy of shear-wave elastography. Furthermore, the intense clinical use of elastography has also identified a high need for standardization, because tissue properties measured with different systems were observed to differ (4). Additionally, in heterogeneous media, the observed wave speed might be determined not only by the average properties of the tissue but also by its boundaries, which can guide the waves and change the observed speed. Proper modeling, such as modeling of arteries as waveguides, allows these effects to be exploited and arterial stiffness characterized on the basis of the observed wave speed (5). In the future, full-wavefield inversion techniques applied to the measured tissue motion might solve this problem, at the cost of increased computational complexity (2).

A clinically emerging method that offers additional tissue contrast is photoacoustic or optoacoustic imaging (6): the thermal expansion of tissue absorbing a short, typically nearinfrared light pulse generates ultrasound that can be detected by the transducers of ultrasound scanners and reconstructed into images showing the optical absorption of tissues. Sweeping through different infrared wavelengths allows multispec- tral imaging with the prospect of tissue characterization and improved diagnosis. Recently, the first clinical applications of photoacoustics were demonstrated as functional overlays on ultrasound images: a dedicated breast scanner showed differences in optical absorption between benign and malignant lesions in the breast (7), and a handheld portable clinical ultrasound system with integrated diode lasers was successfully used for the early detection of rheumatoid arthritis (8). Soon, clinical translation of intravascular photoacoustics combined with intravascular ultrasound is also expected, bringing with it the capability of imaging lipids in atherosclerotic plaques to assess their vulnerability (9).

\section{CONTRAST-ENHANCED ULTRASOUND IMAGING (CEUS)}

For many years, CEUS has been established in clinical routine for characterization of suspected lesions in many organs, especially the liver, and for detecting cardiovascular abnormalities (e.g., using echocardiography). In cardiovascular imaging, microbubbles are used predominantly to enhance the signal in the vessel lumen and to visualize stenoses and aneurysms (10). Especially for the detection of endoleaks after endovascular aneurysm repair, CEUS showed specificity and sensitivity similar to contrast-enhanced CT (10).

The use of microbubbles to detect sentinel lymph nodes is a relatively novel approach. The microbubbles are injected periareolarly and then travel to regional lymphatic sites via subcutaneous lymphatic drainage after tissue massage (Fig. 1C) (11). Shimazu et al. claimed that the rate for detecting the primary draining lymph node is $98 \%$ and that the accuracy of CEUS may even be higher than that of standard clinical methods (injection of blue dye or radiotracer) (11).

Besides using microbubbles to enhance tissue contrast, functional information about tissue vascularization can be obtained using parameters from time-intensity curves such as area under the curve, peak enhancement, and time to peak from bolus injection curves or blood velocity from disruption-replenishment curves (Fig. 1D). Quaia et al. (12) evaluated several parameters resulting from bolus injection time-intensity curves for their capability to differentiate a response to pharmacologic treatment by patients with clinically active Crohn disease. Here, CEUS clearly differentiated responders from nonresponders and was an objective measure to assess therapy response (12). Thus, 


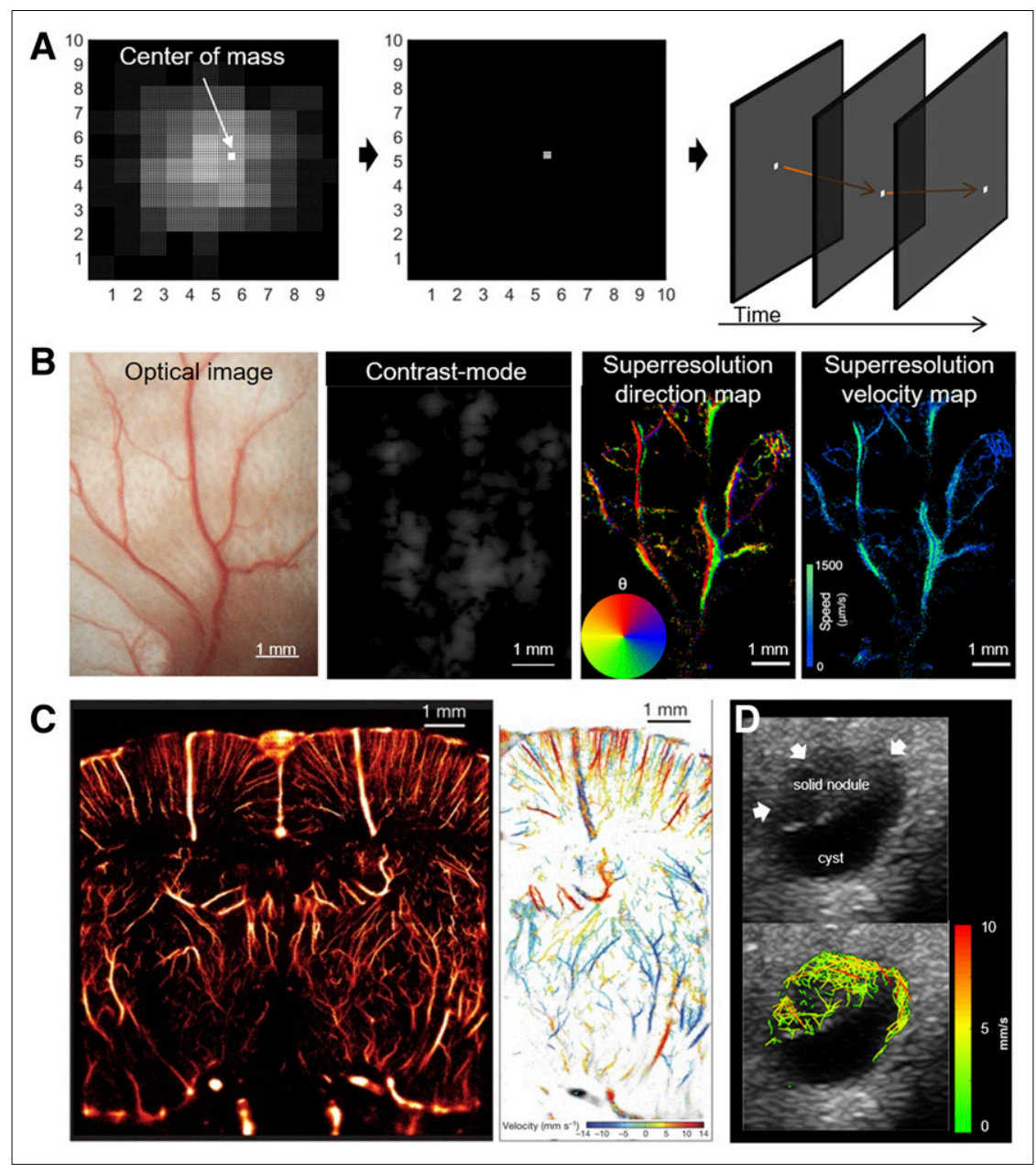

FIGURE 2. Superresolution ultrasound imaging. (A) The concept of many superresolution ultrasound imaging techniques is to identify individual microbubbles (which are presented by system's point-spread function) within imaging plane and determine their center of mass. Center of mass, having approximate size of actual individual microbubble, is plotted onto new image and tracked over time to generate functional superresolution images. (B) In example for mouse ear, fine blood-vessel network can hardly be displayed in contrast mode because of low resolution of ultrasound device. However, superresolution images, calculated from same contrast-mode data, reveal structure of vascular network as well as blood flow direction and velocity in individual vessels. (Reproduced with permission of (17).) (C) In example for mouse brain, superresolution imaging proves its ability to depict minute vascular structures and corresponding blood flow velocities in them. (Reproduced with permission of (18).) (D) Besides promising preclinical data, initial clinical data from thyroid nodule show clinical translatability of superresolution ultrasound. Interconnected microbubble tracks can be localized in solid nodule (arrows).

the authors suggested CEUS as a potential alternative to MRI and CT for grading Crohn disease activity (12).

To assess the reproducibility and reliability of CEUS biomarkers, Lassau et al. (13) performed a clinical study on hepatic and extrahepatic tumors. Particularly, area under the curve and area under the washout proved to be highly reliable (13). It is expected that new imaging procedures, refined postprocessing techniques, and new 3D transducers will further improve the acceptance of CEUS as an imaging technique complementary to MRI and CT. In a recent first-in-humans pilot study, El Kaffas et al. showed that 3D CEUS using a matrix array transducer is superior to conventional 2-dimensional scanning techniques and claimed a higher repeatability of the measurements and a reduced sampling error resulting from tumor tissue heterogeneity (14).

\section{SUPERRESOLUTION ULTRASOUND IMAGING}

Similar to the 2014 Nobel Prize in chemistry, which was awarded for breaking the diffraction barrier to generate superresolution microscopy images, ultrasound imaging is striving for superresolution to analyze the microvasculature of tissues.

Pioneering work on generating superresolution images by tracking the path of microbubbles in vivo was published by Siepmann et al. in 2011 (15). The concept comprised the detection of individual microbubbles to generate superresolution images by plotting their centroid positions and tracking their movement over time (Fig. 2A). A while later, other studies showed that this strategy is capable of increasing the resolution of a clinical ultrasound system by a factor of 2.2-5.1 (16). In 2015, the group of Eckersley published the first in vivo data on functional superresolution ultrasound (17). Their image postprocessing algorithm enabled the extraction of flow direction and velocity information from the microvasculature of a mouse ear (Fig. 2B). They could visualize vessels as small as $19 \mu \mathrm{m}$ - a much higher resolution than is achievable with clinical Doppler imaging. Errico et al. further advanced superresolution ultrasound by applying it on experimental devices that record 500 frames/s. Such a high frame rate, and scanning times of up to $10 \mathrm{~min}$, were necessary to track individual microbubbles to obtain exquisite vascular images of the mouse brain (Fig. 2C) (18). Using a different tracking algorithm, the acquisition of superresolution ultrasound was also successfully shown at lower frame rates. However, initial clinical data indicate that this translation is challenging and requires a refined imaging protocol and inplane and out-of-plane motion correction (Fig. 2D) (19). The latter may be addressed by postprocessing data from 3D matrix transducers, which will also enable 3D assessment of vascular architecture in healthy and pathologic tissues.

\section{MOLECULAR ULTRASOUND IMAGING}

Ultrasound imaging with targeted microbubbles has successfully been applied preclinically to investigate molecular 


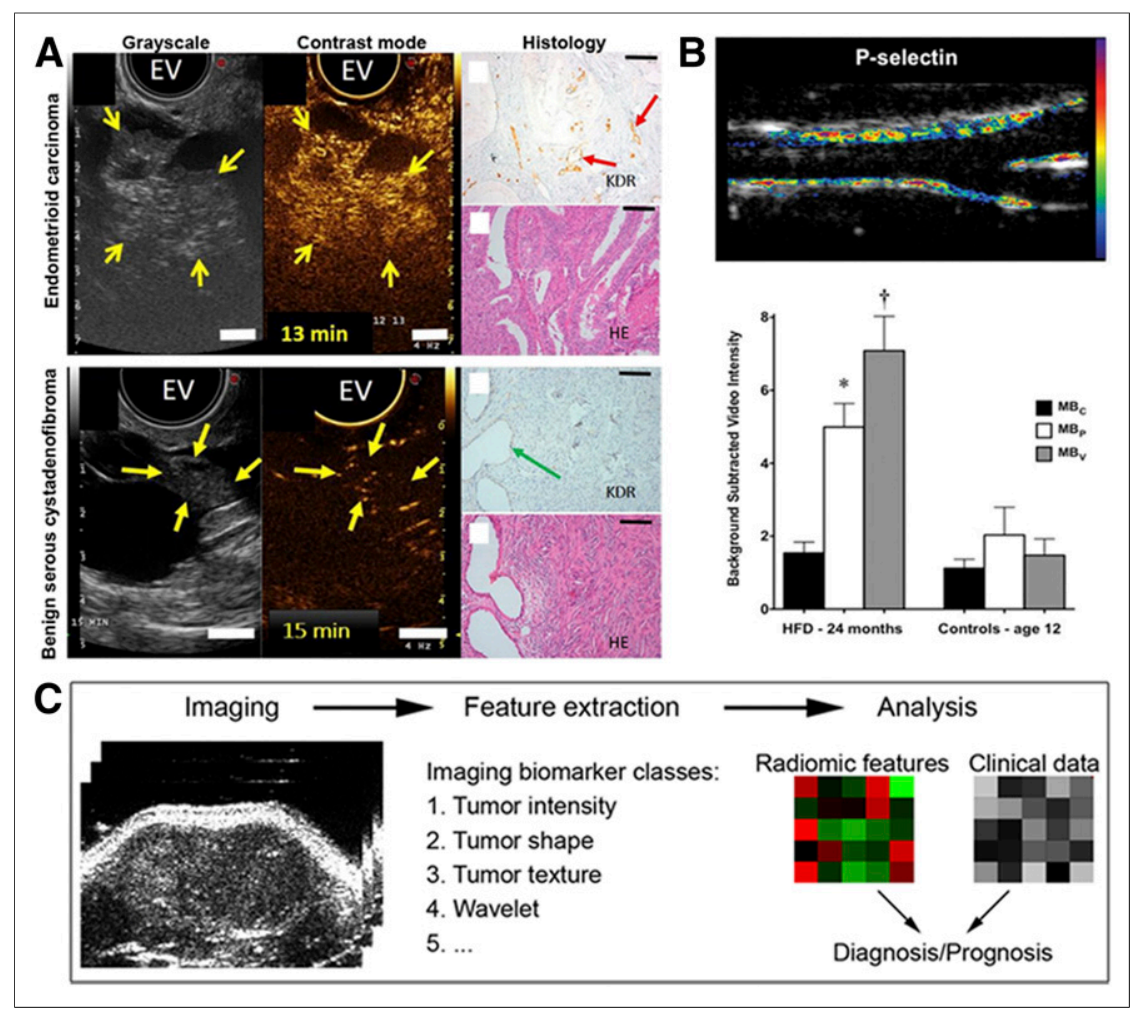

FIGURE 3. (A) Molecular ultrasound imaging with BR55 for characterizing tumor angiogenesis and vascular inflammation detects enhanced VEGFR2 levels in malignant ovarian lesion. Transverse endovaginal (EV) grayscale ultrasound images of right ovaries in 2 patients show both solid (yellow arrows) and cystic lesions. Contrast-enhanced images after intravenous administration of BR55 show strong signal in solid portion of endometrioid carcinoma, whereas only minimal microbubble binding is seen in cystadenofibroma. Corresponding histologic analyses validate strong VEGFR2 expression (red arrows) on endothelium of endometrioid carcinoma and low VEGFR2 expression (green arrow) in cystadenofibroma. Scale bars $=1 \mathrm{~cm}$ for ultrasound images with permission of (21).) (B) Molecular ultrasound imaging with P-selectin and VCAM-1targeted microbubbles detects early inflammation of carotid artery in primates with diet-induced obesity: color-coded molecular ultrasound image showing signals for P-selectin at endothelial surface in primate after 1 y of high-fat diet (HFD) (top), and mean \pm SEM background-subtracted video intensity for P-selectin ( $\left.M_{P}\right)$, VCAM-1 $\left(\mathrm{MB}_{\mathrm{V}}\right)$, and control $\left(\mathrm{MB}_{\mathrm{C}}\right)$ microbubbles in primates exposed to HFD for 24 mo (average age, 12 y) compared with age-matched controls on normal diet (bottom). (Reproduced with permission of (24).) (C) Ultrasound-based radiomics makes use of tomographic medical images to extract quantifiable features from various imaging biomarker classes. Data-mining strategies are used for combined analysis of radiomic features (derived from various imaging biomarker classes) and clinical data to improve diagnosis or prognosis. and $100 \mu \mathrm{m}$ for tissue sections. KDR = kinase insert domain receptor. (Reproduced

ultrasound examinations were well tolerated, and VEGFR2 expression in the malignant breast and ovarian tumors matched well with the molecular ultrasound imaging data (Fig. 3A).

For characterization of inflammation in cardiovascular and gastrointestinal diseases, mainly microbubbles targeting P-selectin, E-selectin, intercellular adhesion molecule 1, and vascular adhesion molecule 1 (VCAM-1) have been used. Transient myocardial ischemia was reliably monitored in rats using clinically translatable dual Pand E-selectin-targeted microbubbles (22). Furthermore, modulated acoustic radiation force-based imaging with P-selectin and VCAM-1-targeted microbubbles enabled rapid detection of inflammation in the abdominal aorta of mice (23). In primates with diet-induced obesity, early inflammation of the carotid artery could be detected by molecular ultrasound imaging targeting VCAM-1 and P-selectin long before any changes in carotid intimamedia thickness were detectable (Fig. 3B) (24). In addition, VCAM-1- and $\alpha_{v} \beta_{3}$-integrin-targeted microbubbles proved promising for monitoring endothelial recovery after vessel injury induced by surgical interventions in mice and pigs, respectively $(25,26)$. Besides monitoring inflammation in cardiovascular disorders, molecular ultrasound imaging, especially with clinically translatable dual-selectin-targeted microbubbles, showed promise in detecting acute colitis and acute phases of chronic inflammatory bowel disease (27).

\section{RADIOMIC ULTRASOUND ANALYSIS}

High-throughput screening and adalterations of the endothelium that occur during pathophysiologic processes in cancer, cardiovascular diseases, and gastrointestinal diseases and in response to therapeutic interventions. In oncology, especially, microbubbles targeting the vascular endothelial growth factor receptor 2 (VEGFR2) have shown a high sensitivity for the in vivo characterization of tumor angiogenesis and the assessment of therapy response. Clinical translation has been achieved with lipopeptide-based microbubbles functionalized with a heterodimer peptide targeting VEGFR2 (BR55). These microbubbles have been applied in patients with prostate cancer, breast cancer, and ovarian tumors $(20,21)$. The vanced pattern-recognition algorithms aim to make unused information contained in ultrasound images available and to identify diagnostically relevant imaging biomarkers and their ideal combinations (Fig. 3C).

Although radiomic studies are emerging for CT and MRI data, hardly any papers describe its application in ultrasound data, most likely because of difficulties in standardizing data acquisition and quantitative data extraction. However, recent studies provide concepts to evaluate and improve the reliability of the parameter maps $(28,29)$. Therefore, it is highly likely that ultrasound data can also be used for radiomic analysis in future. A first proof-of-concept study 


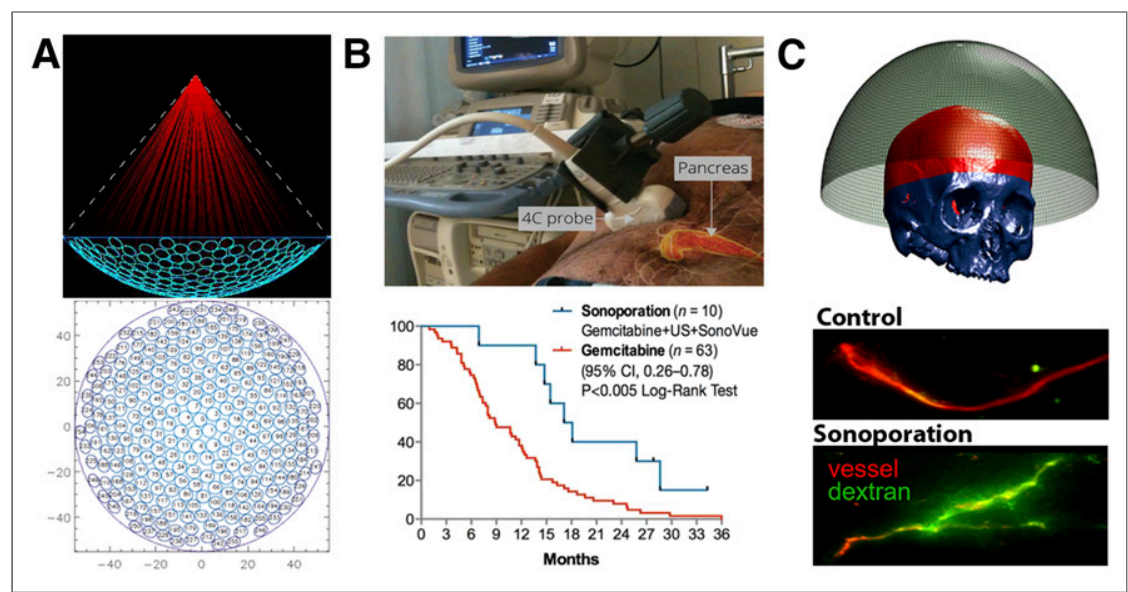

FIGURE 4. (A) Principle of HIFU: sketch illustrates spherically shaped HIFU transducer, with 256 circular transmission elements with converging ultrasound beam path indicated in red, and layout of individual elements in transducer surface. This layout minimizes unused surface between elements and maximizes asymmetry along all directions to reduce unwanted side lobes when steering focal point electronically by appropriate phase changes of electrical signal to transducer elements. (Image courtesy of Charles Mougenot.) (B) Setup and outcome of clinical trial in which pancreatic cancer patients were treated with combination of gemcitabine plus sonoporation. Tumor responses were better than for historical cohort treated with gemcitabine alone. $\mathrm{Cl}=$ confidence interval; SonoVue = sulfur hexafluoride (Bracco); US = ultrasound. (Reprinted with permission of (37).) (C) Sonoporation application to brain: transducer array with 8,907 elements and segmented skull, which allows application of focused ultrasound in brain (top; reprinted with permission of (39)), and effect of sonoporation of healthy mouse brain on extravasation and penetration of prototypic macromolecular drug carrier, 70-kDa fluorescein isothiocyanate (FITC)-dextran (bottom).

could show that radiomic analysis of ultrasound data is possible and can help in the differentiation of different tumor models (30).

\section{HIGH-INTENSITY FOCUSED ULTRASOUND (HIFU)}

HIFU can lead to local tissue heating, cavitation, and radiation forces, which can be used for a variety of therapies such as tissue ablation, image-guided drug delivery, sensitization to radiation therapy, and immune stimulation. Such therapies can be performed with high spatial precision using transducers that are designed specifically for therapeutic purposes and that allow focusing of ultrasound waves deep inside the body into a region about $1 \mathrm{~mm}$ in diameter, depending on the frequency and aperture of the transducer and on the tissue characteristics (Fig. 4A) (31).

HIFU, whether guided by ultrasound or MRI, is a clinically approved technology for treatment of essential tremor, uterine fibroids, and prostate cancer and for palliation of pain in bone metastases. Clinical research is ongoing in many other applications, such as treatment of tumors of the breast, liver, kidney, pancreas, and bone, as well as for obsessive compulsive disorders. Ultrasound and MRI can be used to guide the procedure, with each technique providing its advantages. Ultrasound imaging has a high temporal resolution and can detect and quantify cavitation with high sensitivity. MRI, although expensive and providing a lower temporal resolution, allows accurate temperature mapping and superior image quality.

Recent technologic advances in MR HIFU have led to applications in the human brain. Aberrations due to passage of ultrasound waves through the human skull are corrected using phasedarray HIFU transducers based on highresolution CT scans. For example, for the treatment of essential tremor, part of the subthalamus is ablated with MR HIFU. The patient is awake during the procedure, and the therapeutic effect is immediate (32).

HIFU has long been used for the treatment of prostate cancer. Various systems are in use or under development. A HIFU transducer is inserted in the rectum or urethra and the ablation is guided by either ultrasound or MRI. There is a recent trend toward partial prostate gland ablation, and HIFU may have an advantage over other ablation approaches (33).

HIFU can efficiently induce hyperthermia, which is known to be an excellent radiosensitizer (34). Thus, studies are ongoing to use HIFU in radiotherapy. Hyperthermia has many physiologic effects, such as increased perfusion and oxygenation. The latter is important since oxygen radicals are involved in most chemical reactions triggered by radiotherapy.

Furthermore, the mechanical effects of HIFU can induce the release of antigens that activate dendritic cells. With many drugs approved or under development for immune system stimulation (or inhibition of tumor-associated immune suppression), there is a bright future for HIFU-supported immunotherapy in oncology. Additionally, focused ultrasound was shown to decrease Alzheimer-related plaque development in the brains of animals - an effect that was also tentatively attributed to immune system stimulation (35).

\section{SONOPORATION}

Microbubble-enhanced ultrasound is increasingly used to improve drug delivery to pathologic sites. In this context, multiple approaches can be envisaged, related either to direct drug delivery (i.e., drug entrapment within the microbubble core or shell) or to indirect drug delivery (i.e., coadministration of drugs and microbubbles, Figs. 4B-4D). Clinically, the most progress has been made with the latter approach, in which oscillating microbubbles induce stable or inertial cavitation to promote vascular perfusion, vascular permeability, or tissue penetration.

This process is called sonoporation and was introduced 2 decades ago to describe the ultrasound- and 
microbubble-facilitated transfection of cells with plasmid DNA. Although some people have argued that an alternative name should be given to the combination of ultrasound plus microbubbles to improve drug delivery in vivo (e.g., sonopermeabilization), we propose use of the original terminology with a somewhat extended initial interpretation and application.

In preclinical studies, sonoporation has been shown to improve the delivery of $100-\mathrm{nm}$ liposomes to and into pancreatic tumors (36). The total tumor accumulation of liposomes was increased by up to $100 \%$, and significantly enhanced penetration into the tumor interstitium was observed (Supplemental Fig. 1; supplemental materials are available at http://jnm.snmjournals.org). A clinical proof of concept for sonoporation-enhanced drug delivery was recently provided by Dimcevski et al., who treated pancreatic cancer patients with a combination of gemcitabine, ultrasound, and microbubbles and who achieved a promising median survival of 17.6 mo (vs. 8.9 mo for a historical cohort treated with gemcitabine alone; Fig. 4B) (37).

Extending the applicability of focused ultrasound and sonoporation, the group of Hynynen has developed helmetlike ultrasound transducers (Fig. 4D) that can be used to induce spatially and temporally controlled opening of the blood-brain barrier within MRI scanners $(38,39)$. In 2015, they started the first clinical study, in which the blood-brain barrier is opened in glioblastoma patients to improve doxorubicin delivery. In this context, it seems crucial to involve imaging to monitor the timing and the extent of bloodbrain barrier permeation. This monitoring can be done by coadministering (MRI) contrast agents together with microbubbles and drugs, as well as by administering hybrid microbubbles containing contrast agents within their shell that are released after their destruction to assess vascular permeation (40). In such setups, imaging allows for the real-time assessment of blood-brain barrier opening and helps to maximize the efficacy of the intervention while minimizing toxicity.

\section{CONCLUSION}

For many years, ultrasound was strongly undervalued in clinical practice. Recent achievements have started to uncover its full diagnostic and therapeutic potential. New ultrasound approaches, including refined image postprocessing tools, will enable quantitative multiparametric image analysis, and the increasing use of ultrasound contrast agents provides access to profound vascular characteristics. Here, the clinical introduction of matrix transducers paves the way for true 3D imaging, and superresolution imaging methods will contribute to significantly improved image quality and enhance the number of accessible imaging features. These advantages provide an entry to radiomics analysis, which so far has been mainly the domain of CT and MRI. Together, these innovations will render ultrasound imaging more quantitative and less user-dependent. In addition, therapeutic perspectives for ultrasound are emerging and being evaluated for various tumors and for neurologic and cardiovascular disorders. Among other reasons, this use was enabled by the integration of focused ultrasound devices into MRI scanners, with the latter providing accurate information about lesion size, location, and composition and about temperature control, thus enabling accurate planning and monitoring of interventions. Furthermore, sonoporation-based drug delivery proved promising in preclinical and early clinical studies and stimulated research on new theranostic microbubbles.

Thus, it can be envisaged that new techniques such as elastography, multiparametric imaging, and superresolution imaging will substantially improve the robustness and diagnostic power of ultrasound. Furthermore, therapeutic applications such as HIFU and sonoporation will increase the therapeutic importance of this modality not only in oncology but also in neurology and the cardiovascular field.

\section{DISCLOSURE}

Financial support was provided by the German Research Foundation (KI1072/11-1, KI1072/20-1, LA2937/1-2), the Federal Ministry of Education and Research (BMBF 031A578A), and the European Research Council (ERC Starting Grant 309495: NeoNaNo). No other potential conflict of interest relevant to this article was reported.

\section{REFERENCES}

1. Kiessling F, Fokong S, Koczera P, Lederle W, Lammers T. Ultrasound microbubbles for molecular diagnosis, therapy and theranostics. J Nucl Med. 2012;53:345-348.

2. Hesse MC, Salehi L, Schmitz G. Nonlinear, simultaneous reconstruction of inhomogeneous compressibility and mass density distributions in unidirectional, pulse-echo ultrasound imaging. Phys Med Biol. 2013;58:6163-6178.

3. Lee SH, Chang JM, Kim WH, et al. Added value of shear-wave elastography for evaluation of breast masses detected with screening US imaging. Radiology. 2014;273:61-69.

4. Bamber J, Cosgrove D, Dietrich CF, et al. EFSUMB guidelines and recommendations on the clinical use of ultrasound elastography. Part 1: basic principles and technology. Ultraschall Med. 2013;34:169-184.

5. Astaneh AV, Urban MW, Aquino W, Greenleaf JF, Guddati MN. Arterial waveguide model for shear wave elastography: implementation and in vitro validation. Phys Med Biol. 2017;62:5473-5494.

6. Valluru KS, Willmann JK. Clinical photoacoustic imaging of cancer. Ultrasonography. 2016;35:267-280.

7. Stavros AT, Lavin P, Schoenfeld D, Ulissey MJ. Breast mass classification based on opto-acoustic features using data mining. Presented at: European Congress of Radiology: Annual Meeting of the European Society of Radiology; March 6-10, 2014; Vienna, Austria.

8. van den Berg PJ, Daoudi K, Bernelot Moens HJ, Steenbergen W. Feasibility of photoacoustic/ultrasound imaging of synovitis in finger joints using a point-ofcare system. Photoacoustics. 2017;8:8-14

9. Bourantas CV, Jaffer FA, Gijsen FJ, et al. Hybrid intravascular imaging: recent advances, technical considerations, and current applications in the study of plaque pathophysiology. Eur Heart J. 2017;38:400-412.

10. Schinkel AF, Kaspar M, Staub D. Contrast-enhanced ultrasound: clinical applications in patients with atherosclerosis. Int $J$ Cardiovasc Imaging. 2016;32:35-48.

11. Shimazu K, Ito T, Uji K. Identification of sentinel lymph nodes by contrastenhanced ultrasonography with sonazoid in patients with breast cancer: a feasibility study in three hospitals. Cancer Med. 2017;6:1915-1922.

12. Quaia E, Sozzi M, Angileri R. Time-intensity curves obtained after microbubble injection can be used to differentiate responders from nonresponders among patients with clinically active Crohn disease after 6 weeks of pharmacological treatment. Radiology. 2016;281:606-616. 
13. Lassau N, Coiffier B, Faivre L. Study of intrapatient variability and reproducibility of quantitative tumor perfusion parameters evaluated with dynamic contrast-enhanced ultrasonography. Invest Radiol. 2017;52:148-154.

14. El Kaffas A, Sigrist RMS, Fisher G. Quantitative three-dimensional dynamic contrast-enhanced ultrasound imaging: first-in-human pilot study in patients with liver metastases. Theranostics. 2017;7:3745-3758.

15. Siepmann M, Schmitz G, Bzyl J, et al. Imaging tumor vascularity by tracking single microbubbles. Proc IEEE Int Ultrason Symp. 2011;38:1906-1908.

16. Viessmann OM, Eckersley RJ, Christensen-Jeffries K, et al. Acoustic superresolution with ultrasound and microbubbles. Phys Med Biol. 2013;58:64476458.

17. Christensen-Jeffries K, Brouwning RJ, Tang MX, et al. In vivo acoustic superresolution and super-resolved velocity mapping using microbubbles. IEEE Trans Med Imaging. 2015;34:433-440.

18. Errico C, Pierre J, Pezet S, et al. Ultrafast ultrasound localization microscopy for deep super-resolution vascular imaging. Nature. 2015;527:499-502.

19. Opacic T, Denks S, Theek B, et al. Super-resolution ultrasound bubble tracking for preclinical and clinical multiparametric tumor characterization. bioRxiv website. https://www.biorxiv.org/content/early/2017/10/16/203935. Published October 16, 2017. Accessed February 28, 2018.

20. Smeenge M, Tranquart F, Mannaerts CK, et al. First-in-human ultrasound molecular imaging with a VEGFR2-specific ultrasound molecular contrast agent (BR55) in prostate cancer: a safety and feasibility pilot study. Invest Radiol. 2017;52:419-427.

21. Willmann JK, Bonomo L, Carla Testa A, et al. Ultrasound molecular imaging with BR55 in patients with breast and ovarian lesions: first-in-human results. $J$ Clin Oncol. 2017;35:2133-2140.

22. Hyvelin JM, Tardy I, Bettinger T, et al. Ultrasound molecular imaging of transient acute myocardial ischemia with a clinically translatable P- and E-selectin targeted contrast agent: correlation with the expression of selectins. Invest Radiol. 2014;49:224-235.

23. Wang S, Unnikrishnan S, Herbst EB, et al. Ultrasound molecular imaging of inflammation in mouse abdominal aorta. Invest Radiol. 2017;52:499-506.

24. Chadderdon SM, Belcik JT, Bader L, et al. Proinflammatory endothelial activation detected by molecular imaging in obese nonhuman primates coincides with onset of insulin resistance and progressively increases with duration of insulin resistance. Circulation. 2014;129:471-478.

25. Curaj A, Wu Z, Fokong S, et al. Noninvasive molecular ultrasound monitoring of vessel healing after intravascular surgical procedures in a preclinical setup. Arterioscler Thromb Vasc Biol. 2015;35:1366-1373.
26. Rix A, Fokong S, Heringer S, et al. Molecular ultrasound imaging of $\alpha \mathrm{v} \beta 3$ integrin expression in carotid arteries of pigs after vessel injury. Invest Radiol. 2016;51:767-775.

27. Wang H, Machtaler S, Bettinger T, et al. Molecular imaging of inflammation in inflammatory bowel disease with a clinically translatable dual-selectin-targeted US contrast agent: comparison with FDG PET/CT in a mouse model. Radiology. 2013;267:818-829.

28. Pitre-Champagnat S, Leguerney I, Bosq J, et al. Dynamic contrast-enhanced ultrasound parametric maps to evaluate intratumoral vascularization. Invest Radiol. 2015;50:212-217.

29. Theek B, Opacic T, Möckel D, et al. Automated generation of reliable blood velocity parameter maps from contrast-enhanced ultrasound data. Contrast Media Mol Imaging. 2017;2017:2098324.

30. Theek B, Opacic T, Lammers T, Kiessling F. Radiomic analysis of contrastenhanced ultrasound data. Presented at: European Molecular Imaging Meeting: 12th Annual Meeting of European Society for Molecular Imaging; May 5-7, 2017; Cologne, Germany.

31. Hynynen K, Jones RM. Image-guided ultrasound phased arrays are a disruptive technology for non-invasive therapy. Phys Med Biol. 2016;61:R206-R248.

32. Elias WJ, Lipsman N, Ondo WG, et al. A randomized trial of focused ultrasound thalamotomy for essential tremor. N Engl J Med. 2016;375:730-739.

33. Golan R, Bernstein AN, McClure TD, et al. Partial gland treatment of prostate cancer using high-intensity focused ultrasound in the primary and salvage settings: a systematic review. J Urol. 2017;198:1000-1009.

34. Datta NR, Ordóñez SG, Gaipl US, et al. Local hyperthermia combined with radiotherapy and-/or chemotherapy: recent advances and promises for the future. Cancer Treat Rev. 2015;41:742-753.

35. Leinenga G, Götz J. Scanning ultrasound removes amyloid- $\beta$ and restores memory in an Alzheimer's disease mouse model. Sci Transl Med. 2015;7:278ra33.

36. Theek B, Baues M, Ojha T, et al. Sonoporation enhances liposome accumulation and penetration in tumors with low EPR. J Control Release. 2016;231:77-85.

37. Dimcevski G, Kotopoulis S, Bjånes T, et al. A human clinical trial using ultrasound and microbubbles to enhance gemcitabine treatment of inoperable pancreatic cancer. J Control Release. 2016;243:172-181.

38. Poon C, McMahon D, Hynynen K. Noninvasive and targeted delivery of therapeutics to the brain using focused ultrasound. Neuropharmacology. 2017;120:20-37.

39. Pajek D, Hynynen K. The design of a focused ultrasound transducer array for the treatment of stroke: a simulation study. Phys Med Biol. 2012;57:4951-4968.

40. Lammers T, Koczera P, Fokong S, et al. Theranostic USPIO-loaded microbubbles for mediating and monitoring blood-brain barrier permeation. Adv Funct Mater. 2015;25:36-43. 